\title{
Switching Transients in Electrical Installations of Buildings in Hong Kong
}

\author{
Dr. E. W. C Lo Dr. L. A. Snider Mr. T.T.M Lai \\ Department of Electrical \& Electronic Engineering \\ University of Hong Kong, Hong Kong
}

\begin{abstract}
More and more sensitive electronic and computer based devices or systems are connected to electrical installations of buildings. The measures to ensure a high power quality in the electricity supply network for the satisfactory operation of these equipment is a hot topic in research of electrical engineering. Electrical transients in one of the important issues in power quality. This paper presents the approach and ideas of a project in University of Hong Kong which addresses this problem with particular emphasis on switching transients in typical electrical installations in buildings of Hong Kong.
\end{abstract}

\section{Introduction}

When the electric arc between a pair of circuitbreaker contacts breaks, the full system voltage suddenly appears across the open gap of the breaker, and hence the R-C-L circuit comprising the electrical system. The resultant voltage appearing across the circuit is of high-frequency component superimposed on the normal system voltage. The total voltage is constituting a switching transient, which can be of a magnitude much higher that of normal system voltage. Switching transients propagate along and reflecting back and forth in all other connected conductors of the electrical installation [1]. During this propagation process the magnitudes of the switching transient voltages may be reduced due to attenuation in cables. However, it can also be well "magnified" due to multiple reflections. Therefore all other parts of the electrical installation in the building are subjected to the switching transients, but to different extents.

In modern buildings, with the advancement in electronic areas, more and more delicate electronic devices are installed. They are installed by the users of the buildings (such as: business computer systems, telecommunication equipment) or for control and management of building service equipment (such as: building automation systems, electronic security systems). These devices are highly vulnerable to transient voltages at supply lines. Any damage or loss of data in these devices may result in severe consequence [3].

At the same time, generally, electric power consumption of individual major loads (such as lift motors, air-handling units and chillers of HVAC heating ventilation and air-conditioning - systems) in large modern buildings is increasing. This further pushes the peak voltage of switching transients to higher values. Therefore, even switching transients from normal switching operation (not only limited to fault clearance type switching) are of significant importance.

In The University of Hong Kong, a research project has been started to address the above mentioned problem of switching transients. The aims of the project are:

- to investigate the switching transient environment in electrical installations of typical buildings in Hong Kong,

- to investigate the possible transient impacts on typical equipment,

- and finally to produce a set of guide lines for helping electrical installation design engineers of Hong Kong in the aspect of switching transient protection.

The investigation process includes both mathematical modeling and site measurements. Several typical buildings of different representative usages are located. For electrical installation in each of these buildings, a detailed mathematical model is built for all the major parts of the real installation with the help of a computer simulation software, EMTP. A library of mathematical models for frequently used electrical devices in Hong Kong are also created during the process. Switching schedule would be used in the case studies in modeling in accordance with time schedule. Probability density functions (pdf) of switching are generated for major equipment with irregular or abnormal switching profile. Extensive site measurement program (including whole-day and peak hour measurement) is planned for all the selected buildings to verify the mathematical models. Based on the analysis of both 
the simulated results and site measurements, sensitivity test would be approached for modification of the electrical installation systems to mitigate the switching transient. Finally, effectiveness of these mitigation techniques will be concluded and a set of guide line for switching transient protection will be produced.

As the project has been started only very recently, this paper will introduce the overall approach and the ideas of the project.

\section{Causes of Transients}

The sources of momentary excess voltages (termed as voltage surges or transients) in electrical installations of buildings may be grouped in to the following categories [5]:

- Lightning,

- Switching,

- Faults, and

- Resonance situations.

In this paper, the discussion will concentrate on the switching transients, with some coverage on the lightning transients.

\subsection{Lightning}

The following are typical characteristics associated with lightning flashes [2]:

- $1 \%$ of stokes exceed $200 \mathrm{kA}$

- $50 \%$ of stokes exceed $28 \mathrm{kA}$

- $99 \%$ of stokes exceed $3 \mathrm{kA}$

- $\mathrm{di} / \mathrm{dt}$ exceeded by $1 \%$ of stokes, $200 \mathrm{kA} / \mu \mathrm{s}$

- $\mathrm{di} / \mathrm{dt}$ exceeded by $50 \%$ of stokes, $30 \mathrm{kA} / \mu \mathrm{s}$

- $\mathrm{di} / \mathrm{dt}$ exceeded by $99 \%$ of stokes, $10 \mathrm{kA} / \mu \mathrm{s}$

A typical current waveform of a lightning flash is shown in Fig.1.

Both the large magnitude of the lightning current and its fast $d i / d t$ are causes of transients. When a building is struck by lightning, the large current flow into the earth develops a large voltage between the building structure and lightning protection system. This type of transients are basically resistive in nature and are common mode voltages. When a sharp rising lightning current is flowing through the long down conductors of the lightning protection system, a rapid time varying magnetic field is produced. The varying magnetic field may then induce high transient voltages to nearly electrical wiring via mutual coupling. Due to the long length of down conductors and extensiveness of electrical distribution cables in high rise buildings, the mutual coupling values can be very significant.

In Hong Kong, there are around 40 thunderstorm days per year with about 0.3 to 3 lightning flashes per $\mathrm{km}^{2}$ per year. And the density of high rise buildings in Hong Kong is very dense, hence transients in electrical installation of high rise buildings resulted from lightning flash needs investigation. Survey on actual design parameters of current lightning protection systems of typical buildings Hong Kong is being carried out. Then the next step is simulation of lightning flashes based on the collected data and statistical data of lightning flashes from the Hong Kong Observatory.

\subsection{Switching}

It has been widely known that, when a switch opens in a single-phase a.c. circuit, the recovery voltage may reach twice as high as the normal peak voltage. On the other hand, when a switch closes, the peak current can reach twice the value of the eventual steady-state current. All these are considered as normal voltage and current transients. There are many other situations where much higher voltage and current transients can arise during switching. These are usually referred to abnormal voltage and current transients. Usually, these switching transients are due to the redistribution of stored energy in the circuit inductance and capacitance. They can be single-energy transient, if only one storage parameter ( $\mathrm{L}$ or $\mathrm{C}$ ) is involved. They can also be double-energy transients if both inductance and capacitance are present in the system.

An typical example of abnormal single-energy transients is inrush magnetizing current of an unloaded transformers or motors. The transient may saturate the iron core, hence the magnetizing current increases to many times its normal value, and the current waveform is heavily distorted.

The cable systems for electricity distribution in high rise buildings are long and extensive, the circuit inductance and/or capacitance may be of significant values. These provide the basis for the occurrence of double-energy transients. Typical examples of this type of transients are current chopping and opening a predominantly capacitive circuit.

Current chopping. When interrupting small current by very forcible current interrupting devices (air circuit breaker or some types of fuse) the current $i$ may be forced to zero before the end of a half cycle. This phenomenon is called current chopping. This usually results in a high transient voltage in a highly inductive circuit (e.g. a no-load transformer or a long distribution cable). The situation is illustrated in Fig. 2. The stored energy of the inductance $L$, $\frac{1}{2} L i^{2}$ discharges into the stray capacitance $C$ and converted to stored energy of the capacitance as $\frac{1}{2} C e^{2}$. 
Equating the two energies:

$$
\frac{1}{2} L i^{2}=\frac{1}{2} C e^{2}
$$

It gives a transient voltage:

$$
e=i \sqrt{ }\left(\frac{L}{C}\right)
$$

In the example of Fig. 2 with $L=10 \mathrm{H}, C=1 \mathrm{nF}$, and $i=22 \mathrm{~mA}$, the transient voltage can easily reach $2,190 \mathrm{~V}$. The transient voltage may restrike the arc in the circuit breaker.

Opening a predominantly capacitive circuit. Many buildings are equipped with capacitor banks for automatic power factor correction. These banks are switched in and out quite frequently as loads vary [4]. High voltage transients may occur when switching out these capacitors, as shown in Fig. 3. If the arc goes out at a current zero (point $p$ in Fig. 3 ), the capacitor is charged at peak normal line voltage, $e$. After a half cycle, the line voltage is at its negative peak value and hence the voltage across the contacts of the switch is now $2 e$. This may result a restrike of the arc and situation are then as though $2 e$ were switched on to the circuit. Therefore a oscillatory voltage appears with natural frequency of $\frac{1}{2 \pi \sqrt{L C}}$ and a maximum voltage to earth of $-3 e$. The oscillation may die down due to circuit resistance as shown in the case (b) situation in Fig. 3. Alternatively the arc may extinguish at the first zero current point of the oscillation (i.e. - $3 e$ peak voltage) as shown in case (a) situation of Fig. 3. The capacitor will then remain charged at value $-3 e$. This charge may be retained until the fundamental voltage has gain reached te giving $4 e$ across the switch. A restriking arc may then arise with an oscillatory voltage of $4 e$ giving a maximum of $5 e$ to earth. This process may continue and, theoretically, very high transient voltages may be built up. Obviously, the probability of the various restrikes occurs at exactly the right timing is small. However, it is quite likely to have a transient voltage of more than $3 e$ during switching of capacitor banks. A typical waveform recorded for this type of transient in real situation is shown in Fig. 4.

\section{Transients in Three-phase Circuits}

For three-phase circuits with ground neutral, the treatment is just the same as single phase cases. In a building, in many cases, three phase electricity is distributed to loads with isolated neutral (or grounded through an impedance). The analysis in transient voltage for these cases is more complicated than with grounded neutral, symmetrical component method may be used as for calculating fault currents in asymmetrical fault [6]. The neutral frequency oscillations that constitute the transient are resolved into positive, negative, and zero sequence components.

\section{Switching in Inverters}

In recent years, for improved efficiencies and added flexibility, more and more power inverters are installed in building service plants. They provides VAV (variable air volume) control in HVAC (heating ventilation and air-conditioning) systems, variable speed drive control in pumping systems and variable speed traction control in lift systems, etc. In transient aspect, two main issues of inverters are of concern: shifted natural voltage and high rate of change of voltage. The problem of the two issues is usually not the magnitude of the "transient" voltages, but the continuous existence of nonsinusoidal, but spiky, voltages [7].

The output of an inverter is usually of three-phase three-wire configuration. The output voltages are not sinusoidal, hence the instantaneous neutral voltage, which is defined as the average of the three instantaneous phase voltages, is no longer zero but high pulsating and spiky. In a voltage source inverter drive, the peak neutral shift voltage is the RMS value of the normal line-to-line voltage. The shifted neutral voltage adds stress on the insulation of the connected appliance (usually motor windings) and it also produces high leakage current which may falsely trigger RCCBs (residual current circuit breakers).

The rate of change of voltage $(d v / d t)$ produced by IGBT (insulated gate bipolar transistor) or MOSFET (metal oxide semiconductor field-effect transistor) based inverter is typically higher than $5,000 \mathrm{~V} / \mu \mathrm{s}$ [8]. This high value of $d v / d t$ produce significant high frequency capacitive currents $\left(C_{\text {stroy }} d v / d t\right)$ through equipment insulation, even with a very small stray capacitance $\left(C_{s t r a y}\right)$ in the insulation (again, usually the winding insulation or motor bearings). This leads to high failure rate of the equipment insulation.

\section{Propagation of Transients}

After an electrical transient occurs, it travels through all parts of the electrical installation. All connected electrical appliance in the electrical installation will be attacked by the transient, but at different levels. Due to the high frequency nature of transients, cables of electrical installation can no longer be treated as simple lumped circuits during the analysis of propagation of transients. The lattice diagram has been widely used in analysis pulse propagation in transmission lines. By using this technique, one can easily see that, as illustrated in an example shown in Fig. 5, the crest voltage of a transient may be built up by multiple reflection in the cable. EMTP models on cables of different types and different sizes are built to investigate in 
more details of the propagation behaviour in real situation and non-square type pulses.

\section{Probability Density Functions of Switching}

Finding out the possible magnitudes of transients is only part of the story in investigating the environment of switching transients, another major part is the frequencies of occurrence of these switching transients of various magnitudes. The frequency of occurrence can be expressed as probability density functions (pdf) of switching. In all buildings, there are many different systems to provide the building service of different purposes according to the client requirements and local legal ordinance. To get a more complete picture of the environment of switching transient, the pdfs for all the major building services system should be investigated. In Hong Kong, for a typical commercial building, the followings are the major building service systems of concern in respect to switching transients:

- Chillers and pumps

- Air handling units and primary handling units.

- Lift and escalators.

- Fresh and flush water pumps

- Fire service pumps and sprinkler pumps

- Sewage and drainage pumps

- Lighting

- Capacitor banks for power factor compensation

The methodology in determining the pdfs of these systems are discussed below.

\subsection{Chillers and pumps}

Chillers and chiller pumps are the key components of HVAC systems. The working profile of a chiller and chiller pump depends on the cooling load of the cooling area, usually the whole floor area of the building. Therefore, the cooling load and its energy used through the whole year should first be calculated according to the local weather condition, OTTV (overall thermal transfer value) of the building, usage of the occupied areas and the control algorithm of the HVAC plant. At the same time, sequencing control and time schedule should be considered in determining the timing of the start and stop of the chillers. HVACSim and similar software packages can be used to calculate the cooling energy data. Some of the calculated data are used to compare with measured data collected by Building Management Systems for verification of accuracy.

6.2 Air handling units and primary handling units Air handling units and primary handling units are the components to distribute the air to area served. Similar to chillers and pumps, the working profile of these units depends heavily on the cooling loads of individual areas/zones. Therefore, the time schedule, the cooling load and its energy used has to be determined through the whole year for each area/zone.

\subsection{Lifts and escalators}

Escalators has very few number of switching per day, and usually the timings are known in advance. For the lifts, the number and timing of start and stop during the morning up-peak period and the evening down-peak period can be estimated from statistical calculations based on the floor populations, capacities of lift cars, number of lift cars and car speeds. The inverse S-P method may also be used for these full load periods. Part load with large amount of inter-floor traffic is a more complicated situation. Real time lift traffic computer simulation has to be used to determine the timing of start and stop of the traction motors. Regenerative power have to be considered, as it affects the harmonic and transient currents on the feeders.

\subsection{Fresh and flush water pumps}

The operation of a water pump is dependent on the consumer water flow rate and the effective volume - the volume between the upper limit for stopping the water pump and the lower limit for starting the water pump. With these parameters known, the frequency of water pump operation and duration of each pump operation can be estimated.

\subsection{Fire service pumps and sprinkler pumps}

Fire service pump and sprinkler pumps are essentially operated only during fire situations, testing and maintenance. Therefore, due to the low probability of fire, only the situations of maintenance and testing are counted. The time schedule of maintenance and testing is used as the pdf in this case.

\subsection{Sewage and drainage pumps}

Since there is no meter for sewage and drainage pumps, frequencies of its operation cannot be calculated by metering. However, it can be estimated from flush water flow rate with a factor on the drainage and the know effective volume of the sink.

\subsection{Lighting}

Time schedule for public lighting such as corridor lighting and tenant operating hour for office lighting should be known for determination of the timing of switching on and off. Normal and essential supplies should be separated in time schedule for accurate calculations. In case of using photo cells in controlling the lighting, the daylight lux level for the area should be considered. Software for daylighting calculation is readily available. 


\subsection{Capacitor bank}

Capacitor banks are usually operated in steps and its operation depends on the power factor setting and the current power factor. In order to calculate timing of the operation, the simulation program for harmonics and transients has to communicate with each other.

Through the above mentioned process, one can estimate the magnitude and frequency of occurrence of voltage transients at a particular point in a given electrical installation. Then one can start the investigate of effects of transients on the operation of a particular piece of instrument connected at that point, ways to protect it from the transients, or ways to reduce the magnitude of transients through redesigning some part of the installation.

\section{Impacts on Electronic Equipment}

Today, the electronic equipment connected to electrical installations are:

- De-centralized and proliferation of computerized equipment.

- Increased logic density and processing speeds.

- Change in power supply technology.

All these lead to greater circuit susceptibility under voltage transients..

Transient in the associated electrical installation will cause electronic equipment to have:

- Operation disruption.

- Insulation breakdown

- Component degradation/latent failure.

- Immediate failure.

\section{Conclusions}

To solve the problem of switching transients, one have to starting from their source.

- Identify all the possible source of significant voltage transients in the electrical installation.

- Calculate the magnitude of each type of voltage transients according the load current, the characteristics of current interruption device, the nature of the switching.

- Model the propagation behaviour of each types of voltage transients according to the electrical, physical and electromagnetic characteristics of the distribution cables in the electrical installation.

- Calculate the pdfs of the switching operations of the building service systems according the operating characteristics and control strategy of the systems.

- Finally, voltage transients at typical socket outlet points of the installation can be determined in terms of its magnitude, frequency of occurrence and distribution.
- Therefore, if necessary, appropriate device may be designed to be added on the points to reduce the impact of transients, or modify some of the design parameter of the installation to improve the transient situation in a global manner.

After going through the whole process on some typical buildings in Hong Kong. The project will summarize some general trends which give us some indications on what sort of problems will appear in what sort of typical design configuration or system usage. Finally, the project will generate some guide lines to help the engineers to make a better design of electrical installations in the aspect of reduce transient voltages.

\section{Reference}

[1] Acosta, J and Cornick, K J. "Field investigations into the factors governing the severity of prestriking transients", IEEE Transactions on Energy Conversion, Vol. 2, No.4, March 1987.

[2] British Standard, BS 6651: 1992 - "Code of Practice for Protection of Structures Against Lightning"

[3] Chrysanthou, C. "Statistical models for differential-mode conversion of common-mode impulse voltage measured on telecommunication pairs", IEEE Transactions on Electromagnetic Compatibility, Vol. 38, No. 3, August 1996.

[4] Grebe, T E. "Application of distribution system capacitor banks and their impact on power quality", IEEE Transaction on Industry Applications, Vol. 32, No. 3, May/June 1996.

[5] Grebe, T E. "Power quality and the utility/customer interface", Southcon '94, Orlando, FL, Mar 29-31, 1994.

[6] Greenwood, A. "Electrical transients in power systems", Wiley-Interscience, New York, 1971

[7] Guardado, J L et al. "Calculation of interturn voltages in machine windings during switching transients measured in terminals", IEEE Transactions on Energy Conversion, Vol. 10, No.1, March 1995.

[8] Gupta, B K. "Protection of motors against high voltage switching surges", IEEE Transactions on Energy Conversion, Vol. 7, No.1, March 1992.

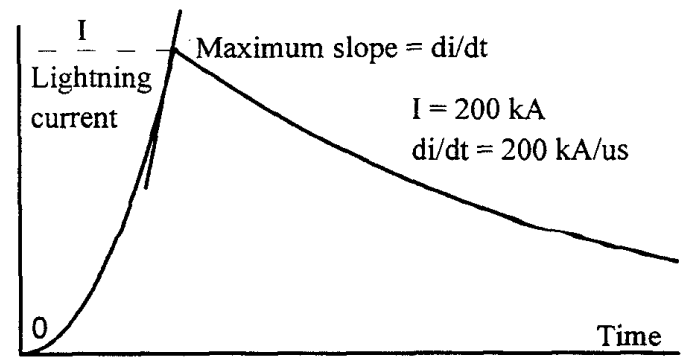

Fig. 1: A typical waveform of lightning flashes 

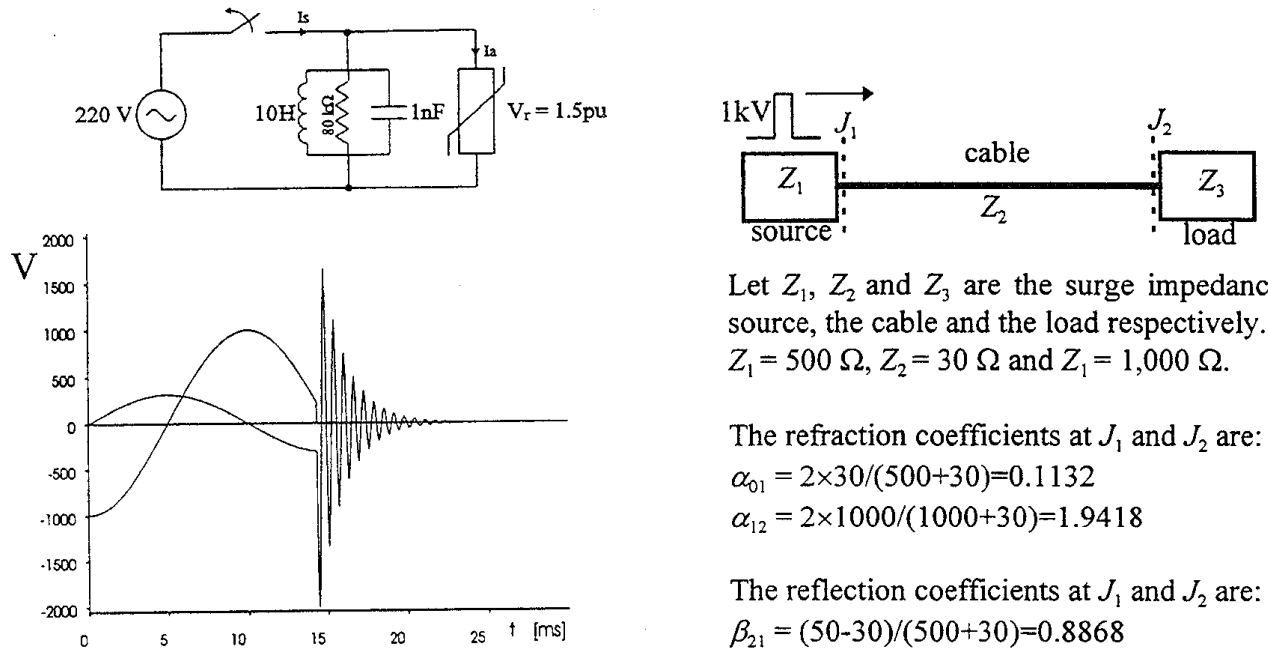

Let $Z_{1}, Z_{2}$ and $Z_{3}$ are the surge impedances of the source, the cable and the load respectively. And Let $Z_{1}=500 \Omega, Z_{2}=30 \Omega$ and $Z_{1}=1,000 \Omega$.

The refraction coefficients at $J_{1}$ and $J_{2}$ are:

$\alpha_{01}=2 \times 30 /(500+30)=0.1132$

$\alpha_{12}=2 \times 1000 /(1000+30)=1.9418$

The reflection coefficients at $J_{1}$ and $J_{2}$ are:

$\beta_{21}=(50-30) /(500+30)=0.8868$

$\beta_{12}=(1000-30) /(1000+30)=0.9417$

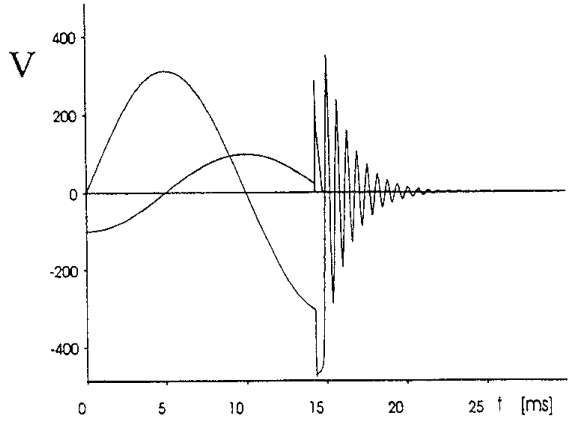

Fig. 2 A simulation results of current chopping

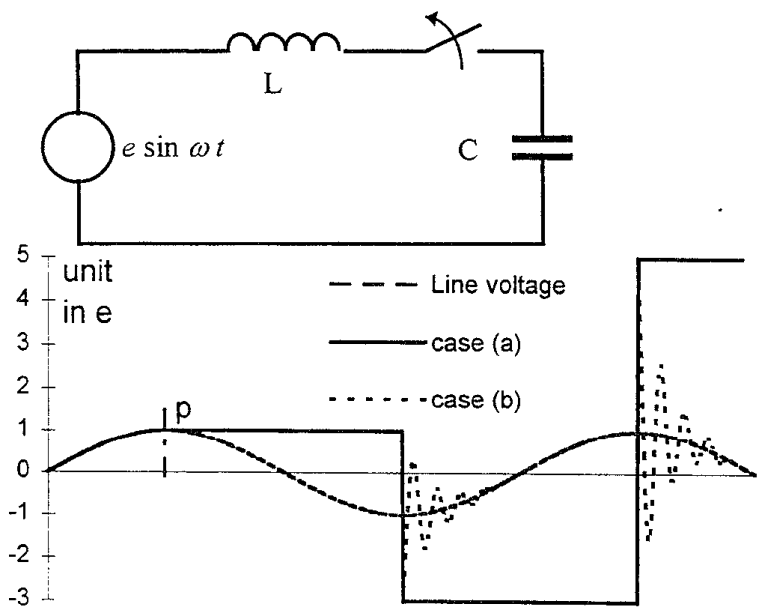

Fig. 3: Simulation result of capacitor bank switching

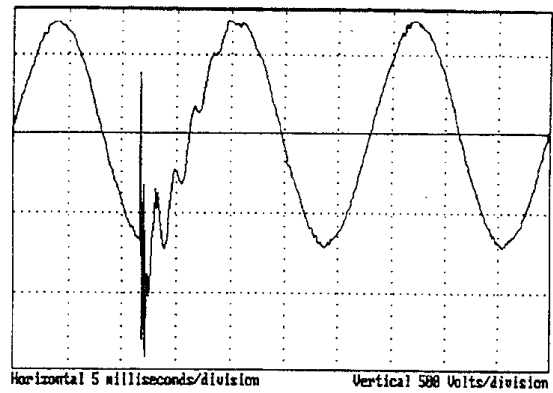

Let $T$ be the transient traveling time through the cable. And assume the width of the transient pulse is longer then $30 \mathrm{~T}$.
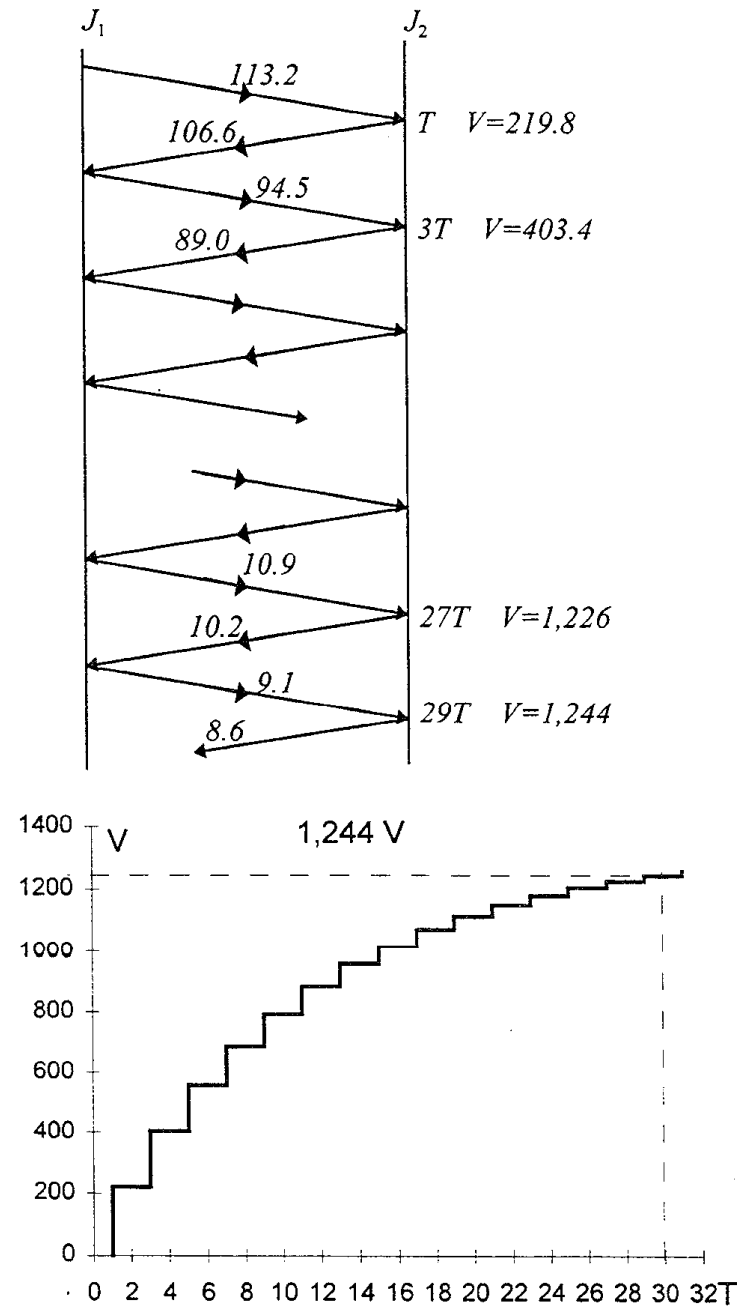

Fig. 4: A recorded waveform of capacitor bank switching 